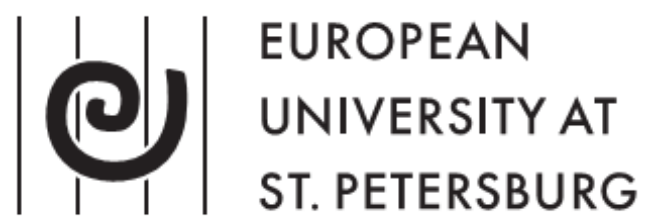

\title{
Maxim Bouev
}

Finding a Valid FX Covariance Matrix in the BS World

Working paper Ec-03/12

Department of Economics

St.Petersburg 2012 


\title{
УДК 330.35 \\ ББК 65.012.2
}

\section{B81}

Bouev M. Finding a Valid FX Covariance Matrix in the BS World. European University at St.Petersburg, Department of Economics. Working Paper Ec-03/12, 28 p.

\begin{abstract}
:
A number of methods has already been proposed for creating a valid correlation matrix in finance. However, such methods do not normally take into account additional restrictions on matrix elements imposed by specific non-arbitrage conditions in some markets, e.g. foreign exchange (FX). I suggest that taking those restrictions, known as triangular relationships, into account can lead to a more efficient method of correction of invalid correlation matrices, at least in FX markets. This paper outlines the steps of the new method.
\end{abstract}

Keywords: correlation matrix, eigenvalue, foreign exchange, triangular relationship, quantitative finance

JEL Classification: C63

\section{Maxim Bouev}

European University at St.Petersburg, 3 Gagarinskaya Street, St.Petersburg, 191187, Russia, mbouev@,eu.spb.ru

The work builds on author's research carried out while working for the RBS bank in London, UK, in 2008-2012.

(C) M. Bouev, 2012 


\section{Finding a Valid FX Covariance Matrix in the BS World}

\section{Introduction}

In finance in general, and in options pricing in particular, the problem of how to specify a correlation matrix occurs frequently. Rebonato and Jäckel (1999) provide a number of examples when it is desirable to alter a given correlation matrix which is for some reason deemed inadequate or inappropriate. The most typical situation is when for a given non-positive semidefinite $n \times n$ matrix $A$ it is necessary to suggest an alternative matrix, $A^{\prime}$, which would in some sense be very close to $A$, but at the same time be positive semi-definite (PSD).

A generally popular solution, without specification of a concrete applied context, has been suggested in Rebonato and Jäckel (1999), where the authors explored a decomposition of matrix $A$ on a hyposphere and also its spectral decomposition. In a nutshell, the essence of their former approach is finding a rather arbitrary PSD target matrix, with elements lying on a unit hyposphere, which guarantees satisfaction of basic restrictions on a correlation matrix, and, which, at the same time, guarantees the best possible fit under some liberally chosen error measure. Their other, spectral decomposition method involves identifying negative eigenvalues of matrix $A$, assigning them new, zero 
values, with some appropriate re-adjustment and re-scaling of corresponding eigenvectors, and, finally, constructing an approximation PSD target matrix. The latter method is, however, not guaranteed to be "optimal" in the sense of error minimisation given some suitable metric.

The two approaches of Rebonato and Jäckel (1999) and their subsequent modifications by Schöttle and Werner (2004) and Roy and Korusoy (2010) went a step further than preceding literature (see inter alia Finger, 1997, and Kupiec, 1998) in that the whole correlation matrix is altered in a controlled fashion, and no slack is allowed for any portions of the matrix. Nevertheless, their methods are not readily applicable in some basic contexts of foreign exchange markets, where, for example, under the assumptions of Black-Scholes (BS) dynamics, there are additional, functional, restrictions on implied correlation coefficients, known as triangular relationships.

In this report I suggest an alternative, simple and fast method, which under conditions of Black-Scholes dynamics in the FX world is guaranteed to produce a valid correlation or covariance matrix. The method preserves all triangular relationships given a complete set of currency pairs. It can be seen, as a specific approach, which, for a given non-positive semi-definite matrix of inadequately specified correlations, finds the best, or nearest, valid matrix of correlations for the complete set of currency markets, evolving according to Black-Scholes 
assumptions. More specifically, the technique employs the implicit function theorem applied to the characteristic polynomial of the covariance matrix, given the non-arbitrage conditions of the Black-Scholes world. With the help of the theorem we ascertain the impact of a marginal change in any variance on the root of the characteristic equation. Should any root be negative, as in the case of non-PSD covariance matrix, the variances can be altered in such a way as to shift the characteristic polynomial in its space, so that it will have only positive roots.

\section{Technical Details}

Before starting the exposition of the new method, let me first present the scope of the specific problem of finding a valid correlation matrix in the FX world driven by Black-Scholes dynamics.

\section{The absence of arbitrage condition and the problem for a complete set of FX markets}

One of the key BS assumptions is the absence of arbitrage in the market. This implies that given an inadequately specified correlation matrix for three currency pairs, for example, EURUSD, GBPUSD, and USDCHF, in order to ensure that the condition of absence of arbitrage holds, it is just not enough to find a valid correlation $3 \times 3$ matrix between the given pairs. This is because any method applied to the given $3 \times 3$ matrix will 
effect a change in the larger correlation matrix $6 \times 6$, which not only includes the correlations between the given 3 currency pairs but also the correlations between three cross markets implied by the original pairs, namely $E U R G B P, E U R C H F$, and $G B P C H F$. It is only the latter, larger $6 \times 6$ matrix, which covers the complete set of markets and, if true, ensures that the zero arbitrage conditions hold. Making a $3 \times 3$ correlation matrix PSD, does not ensure that the larger $6 \times 6$ matrix is $\mathrm{PSD}$, and, in addition, that also might violate the absence of arbitrage conditions in the complete set of markets.

The above logic implies that in the specific applied context of the FX world a risk-, portfolio-manager, or a trader wishing to find a valid correlation matrix have to apply any correction method only to matrices providing correlations for a complete set of markets, which thus, will have dimensions $1,3,6, \ldots$, $\frac{m(m-1)}{2}$, where $m$ is the number of different currencies represented in the set of currency pairs in focus. In other words, all implied cross-markets must not be left out.

\section{An equivalent problem}

Suppose now we have a complete set of $n$ FX markets and a corresponding correlation matrix $R$ of dimension $n \times n$ that we need to make PSD: 


$$
R=\left(\begin{array}{cccc}
1 & \rho_{12} & \ldots & \rho_{1 n} \\
\rho_{21} & 1 & \ldots & \rho_{2 n} \\
\ldots & \ldots & \ldots & \ldots \\
\rho_{n 1} & \rho_{n 2} & \ldots & 1
\end{array}\right)
$$

This problem is equivalent to making PSD a corresponding $n \times n$ covariance matrix, $C$ :

$$
\begin{aligned}
C & =\left(\begin{array}{cccc}
c_{11} & c_{12} & \ldots & c_{1 n} \\
c_{21} & c_{22} & \ldots & c_{2 n} \\
\ldots & \ldots & \ldots & \ldots \\
c_{n 1} & c_{n 2} & \ldots & c_{n n}
\end{array}\right) \\
& =\left(\begin{array}{cccc}
\sigma_{1}^{2} & \rho_{12} \sigma_{1} \sigma_{2} & \ldots & \rho_{1 n} \sigma_{1} \sigma_{n} \\
\rho_{12} \sigma_{2} \sigma_{1} & \sigma_{2}^{2} & \ldots & \rho_{2 n} \sigma_{2} \sigma_{n} \\
\ldots & \ldots & \ldots & \ldots \\
\rho_{1 n} \sigma_{n} \sigma_{1} & \rho_{2 n} \sigma_{n} \sigma_{2} & \ldots & \sigma_{n}^{2}
\end{array}\right)
\end{aligned}
$$

For market practitioners it is generally easier to think about correlations, i.e. direct amendments to matrix $R$, but, at the same time, they do have to make sure that any changes to correlations make sense in terms of volatilities/variances too. Therefore, we prefer to continue considering the equivalent problem of making matrix $C$ positive semi-definite. 
In the BS world correlation coefficients $\rho_{i j}$ are functions of instantaneous variances, namely $\rho_{i j}=\rho_{i j}\left(\sigma_{i}^{2}, \sigma_{j}^{2}, \Sigma_{i j}\right)$, i.e. of variances $\sigma_{i}^{2}$ and $\sigma_{j}^{2}$ and of some set of variances $\Sigma_{i j}$ which is a subset of the full set of possible variances for a given complete set of FX markets, $\Sigma=\left\{\sigma_{1}^{2}, \ldots, \sigma_{n}^{2}\right\}$. In general, $\Sigma_{i j} \subset \Sigma / \sigma_{i}^{2} / \sigma_{j}^{2}$ and will contain either just one element $\sigma_{i / j}^{2}$, i.e. the variance of the cross-pair, if currency pairs $i$ and $j$ have a currency in common, or four elements if currency pairs $i$ and $j$ do not have a currency in common. Moreover, in fact as it is always the case that $\rho_{i j}=\frac{\widetilde{\rho}_{i j}\left(\sigma_{i}^{2}, \sigma_{j}^{2}, \Sigma_{i j}\right)}{2 \sigma_{i} \sigma_{j}}$, where $\widetilde{\rho}_{i j}\left(\sigma_{i}^{2}, \sigma_{j}^{2}, \Sigma_{i j}\right)$ are linear functions of variances, the problem of specification of matrix $C$ becomes a problem of choice of correct variances in the equivalent matrix $\widetilde{C}$ :

$$
\widetilde{C}=\left(\begin{array}{cccc}
\sigma_{1}^{2} & \frac{1}{2} \widetilde{\rho}_{12}\left(\sigma_{1}^{2}, \sigma_{2}^{2}, \Sigma_{12}\right) & \ldots & \frac{1}{2} \widetilde{\rho}_{1 n}\left(\sigma_{1}^{2}, \sigma_{n}^{2}, \Sigma_{1 n}\right) \\
\frac{1}{2} \widetilde{\rho}_{12}\left(\sigma_{1}^{2}, \sigma_{2}^{2}, \Sigma_{12}\right) & \sigma_{2}^{2} & \ldots & \frac{1}{2} \widetilde{\rho}_{2 n}\left(\sigma_{2}^{2}, \sigma_{n}^{2}, \Sigma_{2 n}\right) \\
\ldots & \ldots & \ldots & \ldots \\
\frac{1}{2} \widetilde{\rho}_{1 n}\left(\sigma_{1}^{2}, \sigma_{n}^{2}, \Sigma_{1 n}\right) & \frac{1}{2} \widetilde{\rho}_{2 n}\left(\sigma_{2}^{2}, \sigma_{n}^{2}, \Sigma_{2 n}\right) & \ldots & \sigma_{n}^{2}
\end{array}\right)
$$




\section{A general description of the new method of making covariance matrix PSD}

\section{Eigenvalues as an implicit function}

Suppose matrix $\widetilde{C}$ is not PSD. That implies that at least one of its $n$ eigenvalues, $\lambda_{i}$, is negative. Consider the characteristic equation of the matrix

$$
|\lambda I-\widetilde{C}|=0
$$

In general, the characteristic polynomial on the left hand side of the equation above has the form

$$
f(\lambda)=\lambda^{n}-b_{1} \lambda^{n-1}+\ldots+(-1)^{n} b_{n}
$$

where $b_{1}=\operatorname{Tr}(\widetilde{C})=\sum \widetilde{c}_{i i}$ and $b_{n}=\operatorname{det}(\widetilde{C})$, and $b_{i}$ is the sum of the $i$-rowed diagonal minors of matrix $\widetilde{C}$. This in fact implies that all $b_{i}$ are functions of the elements of matrix $\widetilde{C}$, or, in other words, functions of $n$ variances, $\sigma_{1}^{2}, . ., \sigma_{n}^{2}$. Thus, the characteristic equation of matrix $\widetilde{C}$ defines a set of points such that $f\left(\boldsymbol{\Lambda}, \boldsymbol{\sigma}_{1}^{2}, \ldots, \boldsymbol{\sigma}_{\mathrm{n}}^{2}\right)=0$. 
The function $\Lambda(\cdot)=f\left(\lambda, \sigma_{1}^{2}, . ., \sigma_{n}^{2}\right): R^{n+1} \rightarrow R^{1} \quad$ is continuously differentiable in all its arguments. Moreover, suppose for now - we shall see later when this assumption holds that all elements of the gradient vector $\left(\frac{\partial f}{\partial \lambda}, \frac{\partial f}{\partial \sigma_{1}^{2}}, . ., \frac{\partial f}{\partial \sigma_{n}^{2}}\right)_{\mid\left(\lambda, \sigma_{1}^{2}, ., \sigma_{n}^{2}\right)}$ are not zero. Then, all the conditions of the implicit function theorem are met, and $f\left(\lambda, \sigma_{1}^{2}, \ldots, \sigma_{n}^{2}\right)=0 \quad$ defines an implicit function $\lambda\left(\sigma_{1}^{2}, . ., \sigma_{n}^{2}\right): R^{n} \rightarrow R^{1}$ in the neighbourhood of $\left(\lambda, \sigma_{1}^{2}, \ldots, \sigma_{\mathrm{n}}^{2}\right)$ with the vector of partial derivatives $\left(\frac{\partial \lambda}{\partial \sigma_{1}^{2}}, . ., \frac{\partial \lambda}{\partial \sigma_{n}^{2}}\right)$ at $\left(\lambda, \sigma_{1}^{2}, \ldots, \sigma_{n}^{2}\right)$ given by $\left(-\frac{\partial \Lambda / \partial \sigma_{1}^{2}}{\partial \Lambda / \partial \lambda}, . .,-\frac{\partial \Lambda / \partial \sigma_{n}^{2}}{\partial \Lambda / \partial \lambda}\right)_{\mid\left(\lambda, \sigma_{1}^{2}, ., \sigma_{n}^{2}\right)}$.

\section{Efficient correction to the covariance matrix}

The implicit function $\lambda\left(\sigma_{1}^{2}, . ., \sigma_{n}^{2}\right)$ effectively gives us a way to see how to change elements of matrix $\widetilde{C}$ to induce change in $\lambda$, or in other words, an eigenvalue of $\widetilde{C}$.

Suppose that $\lambda$ is the largest in modulus negative eigenvalue, and that there are no restrictions on which variances $\sigma_{i}^{2}$ can potentially be adjusted. Re-setting the $\lambda$ to a new value $\lambda^{\prime}$ (which can be either zero or a tiny positive $\varepsilon$ ), like in 
Rebonato and Jäckel (1999), can be achieved through corresponding changes in variances $\sigma_{i}^{2}$. In particular, starting with $\widetilde{C}$ we can iteratively adjust $\sigma_{i}^{2}$ following the classic Newton-Raphson method to induce convergence to the desired value $\boldsymbol{\lambda}^{\prime}$ and new matrix $\widehat{C}$ :

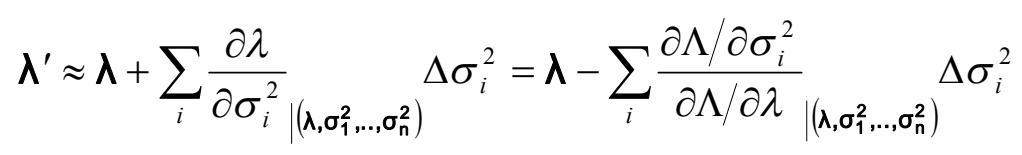

The only problem here is to chose $\Delta \sigma_{i}^{2}$ optimally in some sense. This is achieved by introducing some measure for the deviation $\widehat{C}-\widetilde{C}$, and solving a constrained minimisation problem. For example, as in Rebonato and Jäckel (1999) one can minimise the sum of squares of the elements on the difference matrix $\widehat{C}-\widetilde{C}$. The optimisation programme is then:

$$
\begin{aligned}
& \min _{\Delta \sigma_{1}^{2}, ., \Delta \sigma_{n}^{2}} \sum_{i}\left(\Delta \sigma_{i}^{2}\right)^{2} \\
& \text { s.t. } \boldsymbol{\Lambda}-\sum_{i} \frac{\partial \Lambda / \partial \sigma_{i}^{2}}{\partial \Lambda / \partial \lambda}{ }_{\mid\left(\lambda, \sigma_{1}^{2}, ., \sigma_{n}^{2}\right)} \Delta \sigma_{i}^{2} \approx \lambda^{\prime} .
\end{aligned}
$$


After setting up a Lagrangian, writing down F.O.C., it is easy to see that the optimal solution must satisfy

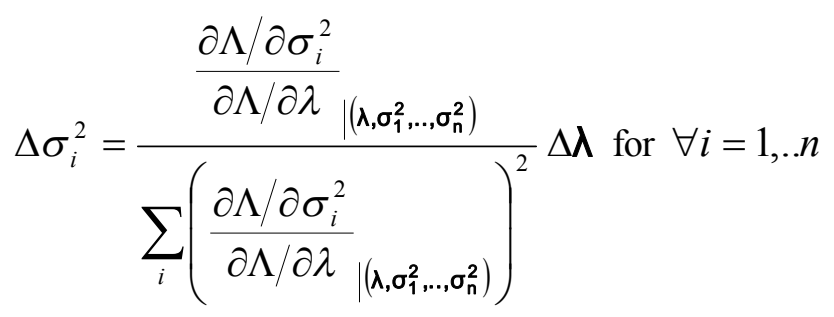

where $\Delta \lambda=\lambda^{\prime}-\lambda$.

In particular, in the situation where one can vary only one variance $k$ to arrive at a PSD matrix $\widehat{C}$, the optimal change must satisfy:

$$
\Delta \sigma_{k}^{2}=\frac{\Delta \Lambda}{\left.\frac{\partial \Lambda / \partial \sigma_{k}^{2}}{\partial \Lambda / \partial \lambda}\right|_{\left.\mid \lambda, \sigma_{1}^{2}, \ldots, \sigma_{n}^{2}\right)}} .
$$

Finally, if the risk manager wants to change any one variance in matrix $\widetilde{C}$, she can choose either the one requiring the minimum absolute change for the same adjustment of eigenvalue $\Delta \lambda$ :

$$
\min _{k}\left|\Delta \sigma_{k}^{2}\right|=\min _{k}\left\{\left|\frac{\Delta \lambda}{\frac{\partial \Lambda / \partial \sigma_{k}^{2}}{\partial \Lambda / \partial \lambda}{ }_{\left(\lambda, \sigma_{1}^{2}, \ldots, \sigma_{n}^{2}\right)}}\right|\right\},
$$


or the one requiring a minimum relative change:

$$
\min _{k}\left|\frac{\Delta \sigma_{k}^{2}}{\sigma_{k}^{2}}\right|=\min _{k}\left\{\left|\frac{\frac{\Delta \lambda}{\sigma_{k}^{2}}}{\frac{\partial \Lambda / \partial \sigma_{k}^{2}}{\partial \Lambda / \partial \lambda} \mid\left(\lambda, \sigma_{1}^{2}, ., \sigma_{n}^{2}\right)}\right|\right\} .
$$

Notice that in the latter case one effectively chooses the variance with the greatest elasticity of change with respect to eigenvalue $\lambda$.

\section{Summing-up: main steps of the method}

Thus, the main steps of the method are as follows:

1. starting with non-PSD matrix $\widetilde{C}$, find its characteristic polynomial $\Lambda(\cdot)=f\left(\lambda, \sigma_{1}^{2}, . ., \sigma_{n}^{2}\right) ;$

2. find eigenvalues of the matrix either by finding roots of the characteristic polynomial directly (which is easily done in certain cases discussed below) or by any other method, such as, e.g. Jacobi (see Press et al., 2007);

3. determine the largest by modulus negative eigenvalue $\boldsymbol{\lambda}$ and compute partial derivatives of the implicit function $\lambda\left(\sigma_{1}^{2}, . ., \sigma_{n}^{2}\right)$ given the eigenvalue $\lambda$ and original variances $\sigma_{1}^{2}, \ldots, \sigma_{\mathrm{n}}^{2}$; 
4. choose the variances you want to change, and adjust them by an optimal amount $\Delta \sigma_{i}^{2}$ so as to reach the desired zero or positive eigenvalue $\lambda^{\prime}$;

5. find new eigenvalues for the adjusted matrix $\widehat{C}$, and repeat steps 1-4 if necessary.

\section{Pros and Cons}

The method just described uses the fact that in the FX BS world properties of the covariance matrix depend solely on the values of its elements on the main diagonal, i.e. variances $\sigma_{i}^{2}$. The off-diagonal elements are functions $\widetilde{\rho}_{i j}\left(\sigma_{i}^{2}, \sigma_{j}^{2}, \Sigma_{i j}\right)$ of the elements on the main diagonal. Thus, to alter properties of $\widetilde{C}$ one has to correct all, or some variances $\sigma_{i}^{2}$. It does not matter for the eventual outcome whether or not there are any restrictions as to which variances can be altered, and which must be kept constant: fixed variances just become parameters in the characteristic polynomial $\Lambda(\cdot)$, but not its arguments, while the remaining variances can be changed.

By introducing functions $\tilde{\rho}_{i j}\left(\sigma_{i}^{2}, \sigma_{j}^{2}, \Sigma_{i j}\right)$, and through varying variances $\sigma_{i}^{2}$ directly, one also ensures that in the BS world the absence-of-arbitrage conditions are met across a complete set of FX markets. 
Moreover, given functions $\widetilde{\rho}_{i j}\left(\sigma_{i}^{2}, \sigma_{j}^{2}, \Sigma_{i j}\right)$, the derivatives $\frac{\partial \Lambda / \partial \sigma_{i}^{2}}{\partial \Lambda / \partial \lambda}$ can be derived analytically, providing for an extremely fast computation of the adjustments in the Newton-Raphson method.

Another advantage of the approach is that by choosing the largest in absolute value negative eigenvalue $\lambda$, and by altering it, one shifts the characteristic polynomial in its space in the most efficient way, so as to ensure that all its roots are to be nonnegative.

The method, however, can potentially run into some difficulties if the eigenvalue $\boldsymbol{\lambda}$ is "too far" from zero, and the linear approximation of the Newton-Raphson method is too crude to cater for fast convergence. Moreover, if the original matrix $\widetilde{C}$ does have zero eigenvalues then the method might fail to "jump" over the zero barrier, and not only "stick" to the zero eigenvalue but fail completely - this is because the conditions of the implicit function theorem, namely the absence of zero elements in the gradient vector $\left(\frac{\partial f}{\partial \lambda}, \frac{\partial f}{\partial \sigma_{1}^{2}}, . ., \frac{\partial f}{\partial \sigma_{n}^{2}}\right)_{\mid\left(\lambda, \sigma_{1}^{2}, \ldots, \sigma_{n}^{2}\right)}$, will not be met. One will have to somehow separate the zero eigenvalues away, and perform the method on a reduced characteristic polynomial, having the same roots as the original one, save for the zero roots. 
The latter problem of the existence of zero eigenvalues is vital in the context of a complete set of FX markets in the BlackScholes world. It is possible to show that absence-of-arbitrage conditions imply that the corresponding covariance matrix will always have zero eigenvalues. Thus, to make sure that the method never fails, one always has to reduce the dimension of the characteristic polynomial. In the next section we demonstrate how to do that for the case with $m$ equal to 3 and 4 , where, as before, $m$ is the number of different currencies represented in the set of currency pairs. In other words, this is a common situation of pricing FX deals on two or three currency pairs with a currency in common. When all crosses are taken into account, the corresponding covariance matrices for the complete set of FX markets will be of dimension $3 \times 3$ and $6 \times 6$, correspondingly.

\section{Application of the method to a $3 \times 3$ covariance matrix}

This case corresponds to a situation when we have two currency pairs with a currency in common, and a cross obtained from the first two pairs either by their division on one another, by multiplication, or by taking an inverse of their product. How exactly the cross is obtained depends on the rules of quotation applied in the market. However, the remarkable fact is that in the Black-Scholes world the resulting characteristic polynomial of the covariance matrix is always the same. 
Consider the covariance matrix

$$
\widetilde{C}_{3}=\left(\begin{array}{ccc}
\sigma_{1}^{2} & \frac{1}{2} \widetilde{\rho}_{12} & \frac{1}{2} \widetilde{\rho}_{13} \\
\frac{1}{2} \widetilde{\rho}_{12} & \sigma_{2}^{2} & \frac{1}{2} \widetilde{\rho}_{23} \\
\frac{1}{2} \widetilde{\rho}_{13} & \frac{1}{2} \widetilde{\rho}_{23} & \sigma_{3}^{2}
\end{array}\right),
$$

where $\widetilde{\rho}_{i j}=\widetilde{\rho}_{i j}\left(\sigma_{i}, \sigma_{j}, \sigma_{i / j}\right)=(-1)^{\delta_{i j}}\left(\sigma_{i}^{2}+\sigma_{j}^{2}-\sigma_{i / j}^{2}\right)$. The Kronecker delta $\delta_{i j}=0$ if the common currency comes on the same side in the market quotation of currency pairs, as, for example, in EURUSD, and GBPUSD, and $\delta_{i j}=1$ if it does not, as in EURUSD and USDCHF.

Proposition 1. For any triangle of currency pairs, $i, j$, and $k=i / j$, and correlation functions $\widetilde{\rho}_{i j}\left(\sigma_{i}, \sigma_{j}, \sigma_{i / j}\right)$, defined as above, with currency pairs $i$ and $j$ being the "drivers", and currency pair $k$ being their cross, the following equality always holds:

$$
(-1)^{\delta_{i j}}(-1)^{\delta_{i k}}(-1)^{\delta_{j k}}=-1
$$

Proof: via Ito’s lemma for long-normal FX processes. 
Using the above preposition it is easy to find that regardless of particular market quotations used for FX rates, the characteristic polynomial for matrix $\widetilde{C}_{3}$ always is

$$
\begin{aligned}
\Lambda(\cdot) & =\lambda^{3}-\lambda^{2}\left(\sigma_{1}^{2}+\sigma_{2}^{2}+\sigma_{3}^{2}\right) \\
& -\frac{3}{4} \lambda\left(\sigma_{1}^{4}+\sigma_{2}^{4}+\sigma_{3}^{4}-2 \sigma_{1}^{2} \sigma_{2}^{2}-2 \sigma_{1}^{2} \sigma_{3}^{2}-2 \sigma_{2}^{2} \sigma_{3}^{2}\right) .
\end{aligned}
$$

It is obvious that it will always have a zero root for the complete set of markets in the BS world. To separate this root away, let us consider a reduced polynomial $\widetilde{\Lambda}(\cdot)$, which has the same nonzero roots as the original characteristic polynomial $\Lambda(\cdot)$ :

$$
\begin{aligned}
\tilde{\Lambda}(\cdot) & =\lambda^{2}-\lambda\left(\sigma_{1}^{2}+\sigma_{2}^{2}+\sigma_{3}^{2}\right) \\
& -\frac{3}{4}\left(\sigma_{1}^{4}+\sigma_{2}^{4}+\sigma_{3}^{4}-2 \sigma_{1}^{2} \sigma_{2}^{2}-2 \sigma_{1}^{2} \sigma_{3}^{2}-2 \sigma_{2}^{2} \sigma_{3}^{2}\right) .
\end{aligned}
$$

In particular, if variances in matrix $\widetilde{C}_{3}$ are for some reason misspecified so that it is non-PSD, then it will have a negative eigenvalue $\boldsymbol{\lambda}$, which will be a root of both $\Lambda(\cdot)$ and $\widetilde{\Lambda}(\cdot)$ at the same time. 
The essence of our method is thus to shift the coefficients of $\tilde{\Lambda}(\cdot)$ in such a way, that it has only positive roots. The remaining steps are as follows:

- the non-zero eigenvalues of $\widetilde{C}_{3}$ are straightforward to find, using the formula for the roots of quadratic equation $\widetilde{\Lambda}(\cdot)=0$; it is also straightforward to see that both roots are always real;

- the partial derivatives of $\tilde{\Lambda}(\cdot)$ are

$$
\begin{aligned}
& \frac{\partial \tilde{\Lambda}(\cdot)}{\partial \lambda}=2 \lambda-\left(\sigma_{1}^{2}+\sigma_{2}^{2}+\sigma_{3}^{2}\right), \\
& \frac{\partial \tilde{\Lambda}(\cdot)}{\partial \sigma_{1}^{2}}=-\lambda-\frac{3}{2}\left(\sigma_{1}^{2}-\sigma_{2}^{2}-\sigma_{3}^{2}\right), \\
& \frac{\partial \tilde{\Lambda}(\cdot)}{\partial \sigma_{2}^{2}}=-\lambda-\frac{3}{2}\left(\sigma_{2}^{2}-\sigma_{1}^{2}-\sigma_{3}^{2}\right), \\
& \frac{\partial \tilde{\Lambda}(\cdot)}{\partial \sigma_{3}^{2}}=-\lambda-\frac{3}{2}\left(\sigma_{3}^{2}-\sigma_{1}^{2}-\sigma_{2}^{2}\right) ;
\end{aligned}
$$

- having obtained the partial derivatives and evaluated them at the point of the negative eigenvalue $\lambda$ and the given set of variances, it is then easy to find optimal corrections to variances $\Delta \sigma_{i}^{2}$ according to formulae 
given in the previous section and a favourable objective function;

- a few iterative steps are enough in practice to shift the characteristic polynomial $\widetilde{\Lambda}(\cdot)$ so that it has only positive roots.

\section{Application of the method to a $6 \times 6$ covariance matrix}

This case corresponds to a situation when we have three currency pairs with a currency in common, as well as all the possible crosses, three in total, obtained by dividing-multiplying the triplets on one another. This situation is more interesting than the previous one, because the covariance matrix will include correlations between currency pairs that do not have a currency in common. Where exactly those correlations are located in the covariance matrix will depend on the ordering of currency pairs. Thus, for clarity of exposition let us suppose that the first three currency pairs have a currency in common, they are the so-called "drivers". The fourth pair, the first "cross" is obtained by dividing or multiplying the first driver on the second driver; the fifth pair is obtained by diving or multiplying the first driver on the third driver, and, finally, the last, the sixth, pair is obtained by dividing or multiplying the second driver by the third driver. In such an ordering the currency pairs $\{1,2,4\},\{1,3,5\},\{2,3,6\}$, and $\{4,5,6\}$ will form four currency triangles. The corresponding covariance matrix will look like: 


$$
\widetilde{C}_{6}=\left(\begin{array}{cccccc}
\sigma_{1}^{2} & \frac{1}{2} \widetilde{\rho}_{12} & \frac{1}{2} \widetilde{\rho}_{13} \frac{1}{2} \widetilde{\rho}_{14} & \frac{1}{2} \widetilde{\rho}_{15} & \frac{1}{2} \widetilde{\rho}_{16} \\
\frac{1}{2} \widetilde{\rho}_{12} & \sigma_{2}^{2} & \frac{1}{2} \widetilde{\rho}_{23} \frac{1}{2} \widetilde{\rho}_{24} & \frac{1}{2} \widetilde{\rho}_{25} & \frac{1}{2} \widetilde{\rho}_{26} \\
\frac{1}{2} \widetilde{\rho}_{13} & \frac{1}{2} \widetilde{\rho}_{23} & \sigma_{3}^{2} & \frac{1}{2} \widetilde{\rho}_{34} & \frac{1}{2} \widetilde{\rho}_{35} & \frac{1}{2} \widetilde{\rho}_{36} \\
\frac{1}{2} \widetilde{\rho}_{14} & \frac{1}{2} \widetilde{\rho}_{24} & \frac{1}{2} \widetilde{\rho}_{34} & \sigma_{4}^{2} & \frac{1}{2} \widetilde{\rho}_{45} & \frac{1}{2} \widetilde{\rho}_{46} \\
\frac{1}{2} \widetilde{\rho}_{15} & \frac{1}{2} \widetilde{\rho}_{25} & \frac{1}{2} \widetilde{\rho}_{35} \frac{1}{2} \widetilde{\rho}_{45} & \sigma_{5}^{2} & \frac{1}{2} \widetilde{\rho}_{56} \\
\frac{1}{2} \widetilde{\rho}_{16} & \frac{1}{2} \widetilde{\rho}_{26} & \frac{1}{2} \widetilde{\rho}_{36} \frac{1}{2} \widetilde{\rho}_{46} & \frac{1}{2} \widetilde{\rho}_{56} & \sigma_{6}^{2}
\end{array}\right),
$$

where $\quad \tilde{\rho}_{i j}=\widetilde{\rho}_{i j}\left(\sigma_{i}, \sigma_{j}, \sigma_{i / j}\right)=(-1)^{\delta_{i j}}\left(\sigma_{i}^{2}+\sigma_{j}^{2}-\sigma_{i / j}^{2}\right), \quad$ if currency pairs $i$ and $j$ come from the same triangle, and $\widetilde{\rho}_{i j}=\widetilde{\rho}_{i j}\left(\sigma_{k}, \sigma_{l}, \sigma_{m}, \sigma_{n}\right)=(-1)^{\delta_{i j}}\left(\sigma_{k}^{2}-\sigma_{l}^{2}-\sigma_{m}^{2}+\sigma_{n}^{2}\right)$ if they do not. In particular, in our $6 \times 6$ matrix only correlations $\widetilde{\rho}_{16}$, $\widetilde{\rho}_{25}$, and $\widetilde{\rho}_{34}$ will have the latter form.

As far as the Kronecker deltas, $\delta_{i j}$, are concerned the following rules will apply. As in the case of the $3 \times 3$ matrix, if currency pairs $i$ and $j$ come from the same triangle, $\delta_{i j}=0$ if the common currency in pairs $i$ and $j$ comes on the same side in the market quotation of currency pairs, and $\delta_{i j}=1$ if it does not. 
If currency pairs $i$ and $j$ do not come from the same triangle, the rules for deltas are much trickier to derive. However, it is possible to avoid introducing separate Kronecker deltas for such pairs altogether. In particular, one can prove the following proposition:

Proposition 2. Let currency pairs $i, j, k, l, m$, and $n$ form the following 4 currency triangles: $\{i, k, l\},\{j, k, m\},\{j, l, n\}$, and $\{i, m, n\}$. Then the correlation function between currency pairs $i$ and $j$, coming from different currency triangles will always be:

$$
\widetilde{\rho}_{i j}=\widetilde{\rho}_{i j}\left(\sigma_{k}, \sigma_{l}, \sigma_{m}, \sigma_{n}\right)=(-1)^{\delta_{i k}} \widetilde{\rho}_{j k}+(-1)^{\delta_{i l}} \widetilde{\rho}_{j l} .
$$

Proof: via Ito's lemma for long-normal FX processes.

Using Propositions 1 and 2, it is straightforward to see that, as in the case of a $3 \times 3$ covariance matrix, in the BS world its characteristic polynomial will be the same regardless of all possible combinations of quotations observed for currency pairs in the market: 


$$
\begin{aligned}
& \Lambda(\cdot)=\lambda^{6}-\lambda^{5}\left(\sigma_{1}^{2}+\sigma_{2}^{2}+\sigma_{3}^{2}+\sigma_{4}^{2}+\sigma_{5}^{2}+\sigma_{6}^{2}\right) \\
& -2 \lambda^{4}\left(\begin{array}{l}
\sigma_{1}^{4}+\sigma_{2}^{4}+\sigma_{3}^{4}+\sigma_{4}^{4}+\sigma_{5}^{4}+\sigma_{6}^{4}-\sigma_{1}^{2} \sigma_{2}^{2} \\
-\sigma_{1}^{2} \sigma_{3}^{2}-\sigma_{1}^{2} \sigma_{4}^{2}-\sigma_{1}^{2} \sigma_{5}^{2}-\sigma_{2}^{2} \sigma_{3}^{2}-\sigma_{2}^{2} \sigma_{4}^{2}-\sigma_{2}^{2} \sigma_{6}^{2} \\
-\sigma_{3}^{2} \sigma_{5}^{2}-\sigma_{3}^{2} \sigma_{6}^{2}-\sigma_{4}^{2} \sigma_{5}^{2}-\sigma_{4}^{2} \sigma_{6}^{2}-\sigma_{5}^{2} \sigma_{6}^{2}
\end{array}\right) \\
& +4 \lambda^{3}\left(\begin{array}{l}
\sigma_{1}^{4} \sigma_{6}^{2}+\sigma_{1}^{2} \sigma_{6}^{4}+\sigma_{2}^{4} \sigma_{5}^{2}+\sigma_{2}^{2} \sigma_{5}^{4}+\sigma_{3}^{4} \sigma_{4}^{2}+\sigma_{3}^{2} \sigma_{4}^{4} \\
+\sigma_{1}^{2} \sigma_{2}^{2} \sigma_{4}^{2}-\sigma_{1}^{2} \sigma_{3}^{2} \sigma_{4}^{2}-\sigma_{2}^{2} \sigma_{3}^{2} \sigma_{4}^{2}-\sigma_{1}^{2} \sigma_{2}^{2} \sigma_{5}^{2} \\
+\sigma_{1}^{2} \sigma_{3}^{2} \sigma_{5}^{2}-\sigma_{2}^{2} \sigma_{3}^{2} \sigma_{5}^{2}-\sigma_{2}^{2} \sigma_{4}^{2} \sigma_{5}^{2}-\sigma_{3}^{2} \sigma_{4}^{2} \sigma_{5}^{2} \\
-\sigma_{1}^{2} \sigma_{2}^{2} \sigma_{6}^{2}-\sigma_{1}^{2} \sigma_{3}^{2} \sigma_{6}^{2}+\sigma_{2}^{2} \sigma_{3}^{2} \sigma_{6}^{2}-\sigma_{1}^{2} \sigma_{4}^{2} \sigma_{6}^{2} \\
-\sigma_{3}^{2} \sigma_{4}^{2} \sigma_{6}^{2}-\sigma_{1}^{2} \sigma_{5}^{2} \sigma_{6}^{2}-\sigma_{2}^{2} \sigma_{5}^{2} \sigma_{6}^{2}+\sigma_{4}^{2} \sigma_{5}^{2} \sigma_{6}^{2}
\end{array}\right) .
\end{aligned}
$$

This polynomial will have three zero roots, that need to be excluded. The reduced polynomial $\widetilde{\Lambda}(\cdot)$ is

$$
\begin{aligned}
& \tilde{\Lambda}(\cdot)=\lambda^{3}-\lambda^{2}\left(\sigma_{1}^{2}+\sigma_{2}^{2}+\sigma_{3}^{2}+\sigma_{4}^{2}+\sigma_{5}^{2}+\sigma_{6}^{2}\right) \\
& -2 \lambda\left(\begin{array}{l}
\sigma_{1}^{4}+\sigma_{2}^{4}+\sigma_{3}^{4}+\sigma_{4}^{4}+\sigma_{5}^{4}+\sigma_{6}^{4}-\sigma_{1}^{2} \sigma_{2}^{2} \\
-\sigma_{1}^{2} \sigma_{3}^{2}-\sigma_{1}^{2} \sigma_{4}^{2}-\sigma_{1}^{2} \sigma_{5}^{2}-\sigma_{2}^{2} \sigma_{3}^{2}-\sigma_{2}^{2} \sigma_{4}^{2}-\sigma_{2}^{2} \sigma_{6}^{2} \\
-\sigma_{3}^{2} \sigma_{5}^{2}-\sigma_{3}^{2} \sigma_{6}^{2}-\sigma_{4}^{2} \sigma_{5}^{2}-\sigma_{4}^{2} \sigma_{6}^{2}-\sigma_{5}^{2} \sigma_{6}^{2}
\end{array}\right) \\
& +4\left(\begin{array}{l}
\sigma_{1}^{4} \sigma_{6}^{2}+\sigma_{1}^{2} \sigma_{6}^{4}+\sigma_{2}^{4} \sigma_{5}^{2}+\sigma_{2}^{2} \sigma_{5}^{4}+\sigma_{3}^{4} \sigma_{4}^{2}+\sigma_{3}^{2} \sigma_{4}^{4} \\
+\sigma_{1}^{2} \sigma_{2}^{2} \sigma_{4}^{2}-\sigma_{1}^{2} \sigma_{3}^{2} \sigma_{4}^{2}-\sigma_{2}^{2} \sigma_{3}^{2} \sigma_{4}^{2}-\sigma_{1}^{2} \sigma_{2}^{2} \sigma_{5}^{2} \\
+\sigma_{1}^{2} \sigma_{3}^{2} \sigma_{5}^{2}-\sigma_{2}^{2} \sigma_{3}^{2} \sigma_{5}^{2}-\sigma_{2}^{2} \sigma_{4}^{2} \sigma_{5}^{2}-\sigma_{3}^{2} \sigma_{4}^{2} \sigma_{5}^{2} \\
-\sigma_{1}^{2} \sigma_{2}^{2} \sigma_{6}^{2}-\sigma_{1}^{2} \sigma_{3}^{2} \sigma_{6}^{2}+\sigma_{2}^{2} \sigma_{3}^{2} \sigma_{6}^{2}-\sigma_{1}^{2} \sigma_{4}^{2} \sigma_{6}^{2} \\
-\sigma_{3}^{2} \sigma_{4}^{2} \sigma_{6}^{2}-\sigma_{1}^{2} \sigma_{5}^{2} \sigma_{6}^{2}-\sigma_{2}^{2} \sigma_{5}^{2} \sigma_{6}^{2}+\sigma_{4}^{2} \sigma_{5}^{2} \sigma_{6}^{2}
\end{array}\right) .
\end{aligned}
$$


If variances in matrix $\widetilde{C}_{6}$ are for some reason mis-specified so that it is non-PSD, then it will have a negative eigenvalue $\lambda$, which will be a root of both $\Lambda(\cdot)$ and $\widetilde{\Lambda}(\cdot)$ at the same time. Again, one needs to shift the coefficients of $\widetilde{\Lambda}(\cdot)$ in such a way, that it has only positive roots. The remaining steps are as follows:

- the non-zero eigenvalues of $\widetilde{C}_{6}$ are straightforward to find, using the formulae for the roots of cubic equation $\widetilde{\Lambda}(\cdot)=0$; it can also be proved that the roots are always real;

- the partial derivatives of $\widetilde{\Lambda}(\cdot)$ are

$$
\begin{aligned}
& \frac{\partial \tilde{\Lambda}(\cdot)}{\partial \lambda}=3 \lambda^{2}-2 \lambda\left(\sigma_{1}^{2}+\sigma_{2}^{2}+\sigma_{3}^{2}+\sigma_{4}^{2}+\sigma_{5}^{2}+\sigma_{6}^{2}\right) \\
& -2\left(\begin{array}{l}
\sigma_{1}^{4}+\sigma_{2}^{4}+\sigma_{3}^{4}+\sigma_{4}^{4}+\sigma_{5}^{4}+\sigma_{6}^{4}-\sigma_{1}^{2} \sigma_{2}^{2} \\
-\sigma_{1}^{2} \sigma_{3}^{2}-\sigma_{1}^{2} \sigma_{4}^{2}-\sigma_{1}^{2} \sigma_{5}^{2}-\sigma_{2}^{2} \sigma_{3}^{2}-\sigma_{2}^{2} \sigma_{4}^{2}-\sigma_{2}^{2} \sigma_{6}^{2} \\
-\sigma_{3}^{2} \sigma_{5}^{2}-\sigma_{3}^{2} \sigma_{6}^{2}-\sigma_{4}^{2} \sigma_{5}^{2}-\sigma_{4}^{2} \sigma_{6}^{2}-\sigma_{5}^{2} \sigma_{6}^{2}
\end{array}\right),
\end{aligned}
$$

$$
\begin{aligned}
& \frac{\partial \tilde{\Lambda}(\cdot)}{\partial \sigma_{1}^{2}}=-\lambda^{2}-2 \lambda\left(2 \sigma_{1}^{2}-\sigma_{2}^{2}-\sigma_{3}^{2}-\sigma_{4}^{2}-\sigma_{5}^{2}\right) \\
& +4\left(\begin{array}{l}
2 \sigma_{1}^{2} \sigma_{6}^{2}+\sigma_{6}^{4}+\sigma_{2}^{2} \sigma_{4}^{2}-\sigma_{3}^{2} \sigma_{4}^{2}-\sigma_{2}^{2} \sigma_{5}^{2} \\
+\sigma_{3}^{2} \sigma_{5}^{2}-\sigma_{2}^{2} \sigma_{6}^{2}-\sigma_{3}^{2} \sigma_{6}^{2}+\sigma_{2}^{2} \sigma_{3}^{2} \sigma_{6}^{2}-\sigma_{4}^{2} \sigma_{6}^{2}-\sigma_{5}^{2} \sigma_{6}^{2}
\end{array}\right),
\end{aligned}
$$




$$
\begin{aligned}
& \frac{\partial \tilde{\Lambda}(\cdot)}{\partial \sigma_{2}^{2}}=-\lambda^{2}-2 \lambda\left(2 \sigma_{2}^{2}-\sigma_{1}^{2}-\sigma_{3}^{2}-\sigma_{4}^{2}-\sigma_{6}^{2}\right) \\
& +4\left(\begin{array}{l}
2 \sigma_{2}^{2} \sigma_{5}^{2}+\sigma_{5}^{4}+\sigma_{1}^{2} \sigma_{4}^{2}-\sigma_{3}^{2} \sigma_{4}^{2}-\sigma_{1}^{2} \sigma_{5}^{2} \\
-\sigma_{3}^{2} \sigma_{5}^{2}-\sigma_{4}^{2} \sigma_{5}^{2}-\sigma_{1}^{2} \sigma_{6}^{2}+\sigma_{3}^{2} \sigma_{6}^{2}-\sigma_{5}^{2} \sigma_{6}^{2}
\end{array}\right), \\
& \frac{\partial \tilde{\Lambda}(\cdot)}{\partial \sigma_{3}^{2}}=-\lambda^{2}-2 \lambda\left(2 \sigma_{3}^{2}-\sigma_{1}^{2}-\sigma_{2}^{2}-\sigma_{5}^{2}-\sigma_{6}^{2}\right) \\
& +4\left(\begin{array}{l}
2 \sigma_{3}^{2} \sigma_{4}^{2}+\sigma_{4}^{4}-\sigma_{1}^{2} \sigma_{4}^{2}-\sigma_{2}^{2} \sigma_{4}^{2}+\sigma_{1}^{2} \sigma_{5}^{2} \\
-\sigma_{2}^{2} \sigma_{5}^{2}-\sigma_{4}^{2} \sigma_{5}^{2}-\sigma_{1}^{2} \sigma_{6}^{2}+\sigma_{2}^{2} \sigma_{6}^{2}-\sigma_{4}^{2} \sigma_{6}^{2}
\end{array}\right), \\
& \frac{\partial \tilde{\Lambda}(\cdot)}{\partial \sigma_{4}^{2}}=-\lambda^{2} \\
& -2 \lambda\left(2 \sigma_{4}^{2}-\sigma_{1}^{2}-\sigma_{2}^{2}-\sigma_{5}^{2}-\sigma_{6}^{2}\right) \\
& +4\left(\begin{array}{l}
\sigma_{3}^{4}+2 \sigma_{3}^{2} \sigma_{4}^{2}+\sigma_{1}^{2} \sigma_{2}^{2}-\sigma_{1}^{2} \sigma_{3}^{2}-\sigma_{2}^{2} \sigma_{3}^{2} \\
-\sigma_{2}^{2} \sigma_{5}^{2}-\sigma_{3}^{2} \sigma_{5}^{2}-\sigma_{1}^{2} \sigma_{6}^{2}-\sigma_{3}^{2} \sigma_{6}^{2}+\sigma_{5}^{2} \sigma_{6}^{2}
\end{array}\right),
\end{aligned}
$$$$
\begin{aligned}
& \frac{\partial \tilde{\Lambda}(\cdot)}{\partial \sigma_{5}^{2}}=-\lambda^{2} \\
& -2 \lambda\left(2 \sigma_{5}^{2}-\sigma_{1}^{2}-\sigma_{3}^{2}-\sigma_{4}^{2}-\sigma_{6}^{2}\right) \\
& +4\left(\begin{array}{l}
\sigma_{2}^{4}+2 \sigma_{2}^{2} \sigma_{5}^{2}-\sigma_{1}^{2} \sigma_{2}^{2}+\sigma_{1}^{2} \sigma_{3}^{2}-\sigma_{2}^{2} \sigma_{3}^{2} \\
-\sigma_{2}^{2} \sigma_{4}^{2}-\sigma_{3}^{2} \sigma_{4}^{2}-\sigma_{1}^{2} \sigma_{6}^{2}-\sigma_{2}^{2} \sigma_{6}^{2}+\sigma_{4}^{2} \sigma_{6}^{2}
\end{array}\right),
\end{aligned}
$$ 


$$
\begin{aligned}
& \frac{\partial \tilde{\Lambda}(\cdot)}{\partial \sigma_{6}^{2}}=-\lambda^{2}-2 \lambda\left(2 \sigma_{6}^{2}-\sigma_{2}^{2}-\sigma_{3}^{2}-\sigma_{4}^{2}-\sigma_{5}^{2}\right) \\
& +4\left(\begin{array}{l}
\sigma_{6}^{2}+2 \sigma_{1}^{2} \sigma_{6}^{2}-\sigma_{1}^{2} \sigma_{2}^{2}-\sigma_{1}^{2} \sigma_{3}^{2}+\sigma_{2}^{2} \sigma_{3}^{2} \\
-\sigma_{1}^{2} \sigma_{4}^{2}-\sigma_{3}^{2} \sigma_{4}^{2}-\sigma_{1}^{2} \sigma_{5}^{2}-\sigma_{2}^{2} \sigma_{5}^{2}+\sigma_{4}^{2} \sigma_{5}^{2}
\end{array}\right) ;
\end{aligned}
$$

- having obtained the partial derivatives and evaluated them at the point of the negative eigenvalue $\lambda$ and the given set of variances, it is then easy to find optimal corrections to variances $\Delta \sigma_{i}^{2}$ according to formulae given in the previous section and a favourable objective function;

- a few iterative steps are enough in practice to shift the characteristic polynomial $\widetilde{\Lambda}(\cdot)$ so that it has only positive roots.

\section{Application of the method to covariance matrices of higher dimensions}

Repeating the same steps as for the matrices $3 \times 3$ and $6 \times 6$, one should be able to establish the following results:

1) The characteristic polynomial for the complete set of FX markets in the BS world does not depend on the concrete currency pair quotations used in the market. 
2) The characteristic polynomial will always have zero roots and, thus, its power must be reduced in order for the method of covariance matrix correction to work.

3) The reduced characteristic polynomial will be a degree $(n-1)$ polynomial, where $n$ is the number of "drivers".

4) All roots of the reduced polynomial are real numbers.

\section{References}

Finger, C. (1997) A Methodology for Stress Correlation. In: RiskMetrics Monitor, pp.3-11, Fourth Quarter.

Kupiec, P.H. (1998) Stress Testing in a Value at Risk Framework. Journal of Derivatives, pp. 7-24, Fall.

Press, W.H., Teukolsky, S.A., Vetterling, W.T., and Flannery, B.P. (2007) Numerical Recipes. The Art of Scientific Computing. Cambridge University Press.

Rebonato, R., and Jäckel, P. (1999) The Most General Methodology to Create a Valid Correlation Matrix for Risk Management and Option Pricing Purposes. The Journal of Risk, Winter 1999/2000, 2(2).

Roy, S., and Korusoy, E. (2010) Obtaining a Valid Correlation Matrix of Exchange Rates Using Prioritized Time Series Approach. Mimeo, Indian Institute of Management, Ahmedabad, 
India 380 015, and Global Banking and Markets, Royal Bank of Scotland, Singapore 048503.

Schöttle, K., and Werner, R. (2004) Improving the "Most General Methodology to Create a Valid Correlation Matrix for Risk Management and Option Pricing Purposes". Risk Analysis IV, Management Information Systems, 9, pp. 701-712. 